\title{
Therapeutic options for treatment of human papillomavirus-associated cancers - novel immunologic vaccines: ADXS11-001
}

\author{
Brett Miles ${ }^{1}$, Howard P. Safran ${ }^{2}$ and Bradley J. Monk ${ }^{3 *}$
}

\begin{abstract}
Survival of patients with advanced, recurrent, or metastatic human papillomavirus (HPV)-associated cancer is suboptimal despite the availability of various treatment modalities. The recently developed bacterial vector Listeria monocytogenes $(\mathrm{Lm})$ activates innate and adaptive immune responses and is expected to offer immunologic advantages. Axalimogene filolisbac (AXAL or ADXS11-001) is a novel immunotherapeutic based on the live, irreversibly attenuated $L m$ fused to the nonhemolytic fragment of listeriolysin $O(L m$ - LLO) and secretes the Lm-LLO-HPV E7 fusion protein targeting HPV-positive tumors. Herein are reported the development and recent results of various clinical trials in patients with HPV-associated cervical, head and neck, and anal cancers.
\end{abstract}

Keywords: ADXS11-001, AXAL, Human papillomavirus, HPV-positive cancers, Clinical trials

\section{Introduction}

Persistent human papillomavirus (HPV) infection is currently acknowledged as a direct cause of cervical, anogenital, and oropharyngeal cancers [1], and has been estimated to account for more than $5 \%$ of all cancers globally [2]. More than half of all cancers attributable to infection worldwide are caused by HPV (Table 1) [3]. Moreover, cervical cancer was the first type of cancer officially recognized by the World Health Organization to be attributable to a viral infection.

This review aims to describe current therapeutic options for HPV-associated cancers, with an emphasis on therapeutic cancer vaccines currently being tested in clinical trials, and a particular focus on describing the efficacy and safety of the novel immunogenic compound axalimogene filolisbac (AXAL or ADXS11-001) in patients with HPV-positive cervical, head and neck, and anal cancers.

\footnotetext{
* Correspondence: bradley.monk@usoncology.com

${ }^{3}$ Division of Gynecologic Oncology, Arizona Oncology (US Oncology

Network), University of Arizona College of Medicine, Creighton University

School of Medicine at St. Joseph's Hospital, 2222 E. Highland Ave, Suite 400

Phoenix, AZ 85016, USA

Full list of author information is available at the end of the article
}

\section{Immunobiology of HPV}

HPV belongs to a family of papillomaviruses that are composed of non-enveloped, double-stranded deoxyribonucleic acid (DNA) viruses able to infect the multilayer stratified tissue (eg, human epithelium). HPV is a sexually transmitted, circular virus encoding for 7 early (E1, E2, E4, E5, E6, E7, and E8) and 2 late, structural (L1 and L2) genes [4]. In cervical cancer, upon sexual transmission, HPV infects the basal epithelial cells of the cervical mucosa, leading to intracellular expression of low levels of viral proteins [5]. Viral DNA replicates following infection, and production of viral proteins is enhanced once HPV-infected cells leave the basal layer [6]. Chronic infection is maintained in approximately $10 \%$ of women because of the capacity of HPV to escape host immune surveillance [7]. The molecular mechanism accounting for persistent HPV infection and carcinogenesis involves the integration of viral DNA into the host genome, accompanied by deletion of both early and late HPV genes, namely E2, E4, E5, L1, and L2. The oncogenic potential of HPV is a result of two early viral proteins, E6 and E7. As a result of loss of the transcriptional regulator gene E2, these two oncoproteins are upregulated. The early viral protein E6 binds to the tumor suppressor gene p53, thereby inhibiting apoptosis of HPVinfected cells $[8,9]$. The early viral protein E7 inhibits 
Table 1 Estimated number of HPV-attributable new cancer cases, by anatomic site and gender

\begin{tabular}{|c|c|c|c|c|c|}
\hline \multirow[t]{2}{*}{ Cancer site } & \multirow[t]{2}{*}{ Number of new cases } & \multirow[t]{2}{*}{ Number of cases attributable to HPV } & \multirow[t]{2}{*}{ Attributable fraction, $\%$} & \multicolumn{2}{|c|}{$\begin{array}{l}\text { Number of cases attributable } \\
\text { to HPV by gender }\end{array}$} \\
\hline & & & & Male & Female \\
\hline Cervix uteri & 528,000 & 501,600 & 95 & - & 528,000 \\
\hline Anus & 40,000 & 35,000 & 88 & 17,000 & 18,000 \\
\hline Vagina and vulva & 49,000 & 20,000 & 41 & - & 20,000 \\
\hline Penis & 26,000 & 13,000 & 51 & 13,000 & - \\
\hline Oropharynx & 96,000 & 29,000 & 31 & 24,000 & 6000 \\
\hline Oral cavity and larynx & 358,000 & 16,000 & 4.4 & 12,000 & 4000 \\
\hline Total & 1096000 & 641,000 & 58 & 66,000 & 575,000 \\
\hline
\end{tabular}

HPV human papillomavirus

functionality of the tumor suppressor retinoblastoma product, thus allowing HPV to replicate in previously differentiated epithelial cells $[9,10]$. Formation of complexes between these two viral proteins and the aforementioned tumor suppressor genes disturbs the normal cycle of cell regulation, causes genomic instability, and ultimately leads to neoplasia. A similar biomolecular process is the basis for development of other HPV-associated cancers.

\section{HPV-associated cancers and current therapies} HPV-associated cancers and prevention of HPV infections More than $100 \mathrm{HPV}$ types have been identified to date [11]. Of these, the most frequently encountered highrisk HPV types, $16,18,31$, and 45 , are together responsible for approximately $80 \%$ of all cervical cancer cases [12-14]. HPV-16 and -18 have been identified as the two most prevalent high-risk HPV types and are accountable for approximately 62.6 and $15.7 \%$, respectively, of cervical cancers [15]. Additionally, these two high-risk HPV types are responsible for $80-86 \%$ of vulvar and vaginal cancers, $89-95 \%$ of oropharyngeal cancers, $93 \%$ of anal cancers, and $63-80 \%$ of penile cancers [16].

Two prophylactic vaccines have been developed for prevention of HPV infection: Gardasil (Merck and Co., Inc.), and Cervarix (GlaxoSmithKline Biologicals). The quadrivalent vaccine Gardasil provides immunologic protection against infection with HPV-6, $-11,-16$, and -18 [17], whereas the bivalent vaccine Cervarix provides protection against infection with HPV-16 and -18 [18]. In addition, the nonavalent vaccine Gardasil 9 (Merck and Co., Inc.) has been demonstrated to protect against HPV-6, $-11,-16,-18,-31,-33,-45,-52$, and -58 [19]. However, despite recent advancements within the field of tumor immunology, no therapeutic vaccines for the treatment of HPV-associated cancers are currently available for general use in the clinical setting.

\section{Current therapeutic options for HPV-associated cancers Cervical and vulvar cancers}

Pre-invasive genital tract neoplasia includes cervical intraepithelial neoplasia (CIN), vaginal neoplasia, and vulvar intraepithelial neoplasia (VIN). Current treatment strategies for CIN include minimally invasive therapies, such as loop electrosurgical excision procedure or cryotherapy [20]. These strategies focus on eliminating the HPV-positive precancerous cells, while maintaining cervical integrity and fertility [21].

The estimated number of new cervical cancer cases raises to 528,000 each year [3], with $95 \%$ cases attributable to HPV [22], while from the 49,000 new cases of vulvar and vaginal cancers estimated, $41 \%$ were attributable to HPV [3]. Chances of survival are high when cervical cancer is identified at early stages. (International Federation of Gynecology and Obstetrics [FIGO] stages IA2-IB1). Treatment consists of conization, radical hysterectomy (preferred), radical trachelectomy (for selected patients), or radiation therapy. Locally advanced tumors are generally treated with concomitant chemoradiotherapy that includes a cisplatin-based regimen [23]. Patients with persistent, recurrent, or metastatic cervical cancer have poor survival and increased morbidity caused by renal failure and clinical deterioration. For these patients, the standard of care consists of platinum-based chemotherapy doublets, such as cisplatin and paclitaxel $[24,25]$, usually administered in combination with the humanized monoclonal antibody directed against vascular endothelial growth factor (bevacizumab) [26]. The main treatment modalities of vulvar cancer consist of surgery, for localized disease, or a combination of surgery and radiation (with or without chemotherapy) when nodal metastases are present $[27,28]$. For patients presenting with vaginal cancers, three types of standard treatment are generally used: surgery (e.g., laser surgery, wide local excision, vaginectomy, total hysterectomy), external or internal radiation therapy [29], and systemic or regional chemotherapy [30]. 


\section{Head and neck cancers}

High-risk oncogenic HPVs represent major risk factors for development of head and neck cancers [31]. These are primarily tumors of the oropharynx, specifically the tonsil and base of tongue. An estimated 96,000 new cases of cancer of the oropharynx was recently reported, out of which 29,000 were attributable to HPV [3]. In the United States it has recently been approximated that HPV-related head and neck cancers incidence is likely to surpass that of cervical cancers by the year 2020 [32]. The primary viral etiology of these cancers is HPV-16; however, up to $9 \%$ may be caused by additional serotypes (e.g., HPV-35, HPV-18) [33]. Despite increasing awareness and improved viral detection methods, identification of active disease remains problematic [34]. Patients with early stage disease can be treated with single-modality therapy. Minimally invasive transoral robotic surgery is also being investigated [35]. The most commonly employed therapies for locally advanced head and neck cancers include cisplatin and concurrent radiation. Concurrent epidermal growth factor receptor antibody and radiation is an alternative. More recent treatments for patients with head and neck cancers include targeted therapies, such as the anti-programmed death protein 1 (PD-1) monoclonal antibody nivolumab. In a phase II trial performed in patients with recurrent or metastatic squamous cell carcinoma of the head and neck, the overall survival (OS) of patients treated with nivolumab was higher than OS following standard platinum chemotherapy (7.5 vs 5.1 months). The 6-month progression-free survival (PFS) rate and the response rate of patients treated with nivolumab were also higher than in patients receiving standard therapy (19.7\% vs $9.9 \%$ and $13.3 \%$ vs $5.8 \%$ ), while the occurrence of grade 3 or 4 treatment-related adverse events (TRAEs) was lower following nivolumab treatment (13.1\% vs $35.1 \%)$ [36]. This proof-of-concept phase II trial not only demonstrated the superior efficacy of nivolumab in patients with recurrent or metastatic squamous cell carcinoma of the head and neck, but also underlined the superior safety profile of nivolumab in this patient population.

\section{Anal and penile cancers}

Annually, 24,000 anal and 11,000 penile cancer cases are reported worldwide, with approximately 21,000 and 6500 cases, respectively, associated with HPV [1]. In 2012, these numbers increased to 40,000 for anal cancer and 26,000 for penile cancer, out of which 35,000 and 26,000 respectively, were reported as attributable to HPV [3]. Anal intraepithelial neoplasia (AIN) is generally treated with minimally invasive methods, such as laser ablation or infrared coagulation [37]; excision is reserved for high-grade AIN cases. The standard of treatment for localized anal cancer is concurrent chemoradiotherapy, consisting of concurrent radiation, mitomycin $\mathrm{C}$, and 5-fluorouracil $[38,39]$. Metastatic anal cancer is not curable. While there is no standard-of-care chemotherapy, options can include platinum analogues, taxanes, or antimetabolites. The best options for localized penile cancer consist of surgical treatments (e.g., excision, microsurgery, laser surgery, circumcision) and radiation, used as an adjuvant to surgery. Current treatment options for recurrent or metastatic penile cancer includes taxanes, platinum analogues, and ifosfamide. [40, 41].

Despite the various treatment modalities currently available, survival of patients presenting with one of the aforementioned advanced, recurrent, or metastatic $\mathrm{HPV}$-associated cancers remains poor.

\section{Therapeutic HPV vaccines}

In patients with HPV-associated cancers, the standard functionality of the innate and adaptive immune systems is altered and tolerance or suppression mechanisms develop, capable of blocking or reversing antitumor immune responses [42, 43]. Tolerance mechanisms interfere with various steps of the antigen-presentation process as well as with the antitumoral activity of cluster of differentiation (CD)4-positive and CD8-positive $\mathrm{T}$ cells, thus rendering them nonfunctional. Immune suppression mechanisms include development of suppressive immune cell populations (e.g., regulatory T cells [Tregs], myeloid-derived suppressor cells [MDSCs], tumor-associated macrophages) with protumoral activity. Overall, HPV-induced diseases are associated with a lack of HPV-specific antitumoral immune responses and an excess of immune-suppressive cellular and humoral protumoral responses. Therefore, the main goal of therapeutic vaccines is to induce or greatly improve HPV-specific T-cell based immunity by making use of the constitutively expressed tumorspecific antigens E6 and E7.

Because of their reported efficacy, protein- and peptide-based vaccines are the most common forms of therapeutic HPV vaccines. Their mechanisms of action involve uptake of the peptide antigen and major histocompatibility complex molecules by dendritic cells, and cross-presentation to CD8-positive T cells (Fig. 1). A phase I study performed in patients with end-stage cervical cancer vaccinated with HPV-16 E6, alone or in combination with HPV-16 E7 overlapping long peptides, reported good vaccine tolerability and broad $\mathrm{T}$-cell responses [44]. Several fusion protein-based vaccine formulations containing the oncogene E7 of the high-risk type HPV-16 have been tested in clinical trials in patients with high-grade AIN [45] or CIN [46, 47], as well as in patients with cervical cancer [48], and have demonstrated varying degrees of efficacy. 


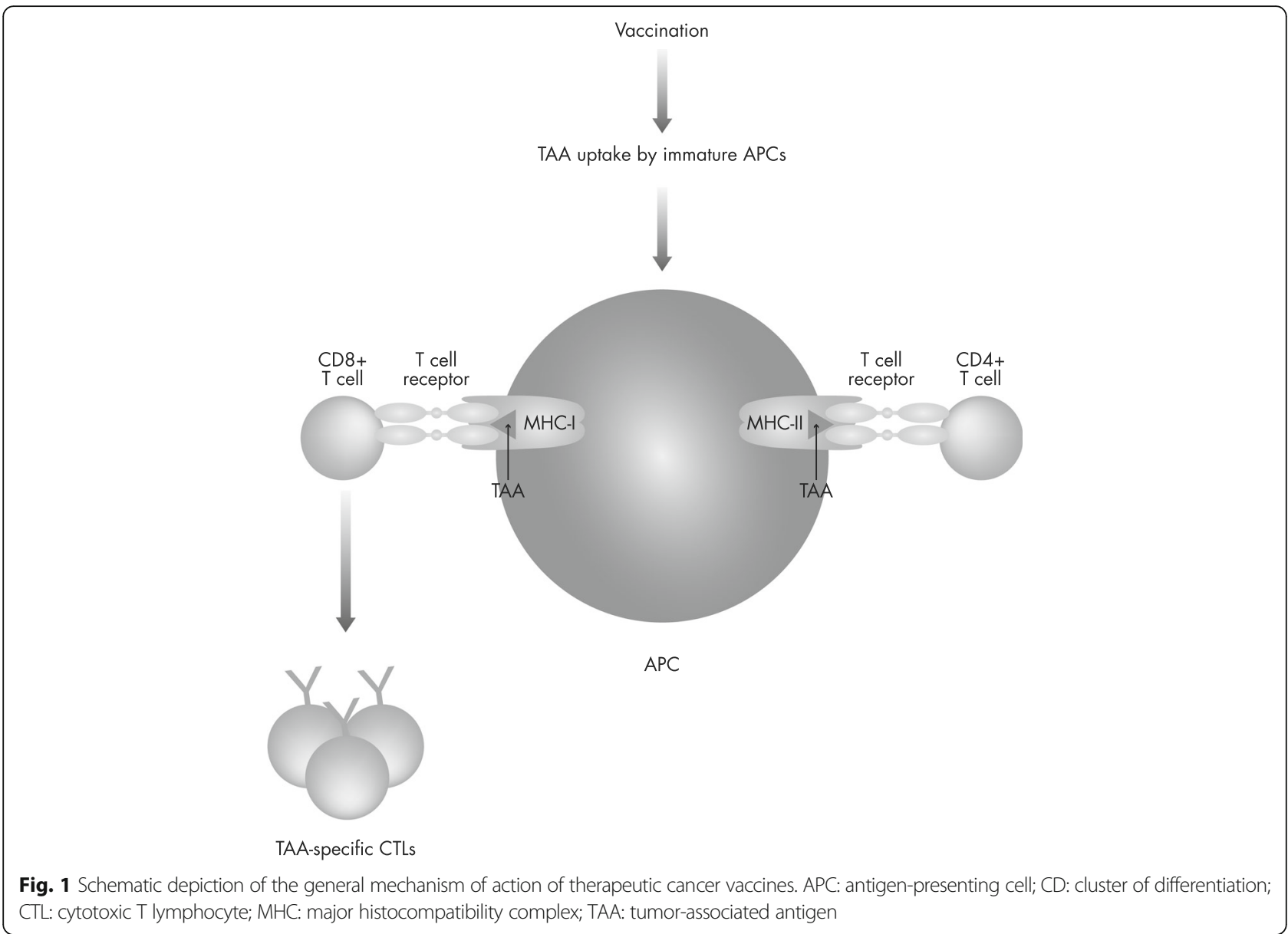

Nucleic acid and whole cell vaccines represent other potential immunotherapeutic strategies tested in clinical trials. A DNA vaccine containing E7 DNA fused with the heat shock protein 70 tested in patients with CIN grade $2 / 3$ was reported to be safe, but induced only lowfrequency E7-specific T-cell responses [49]. Similarly, autologous dendritic cells pulsed with HPV-16 or HPV-18 E7 recombinant proteins in patients with stage IB-IIA cervical cancer [50] or patients with late-stage disease [51] led to antigen-specific serologic responses of varying degrees; however, there was no sustained limitation on tumor burden. All of the aforementioned vaccine formulations were well tolerated and induced antigenspecific cell-mediated immunity to varying degrees. However, the rates of lesion regression observed within these studies were lower than $50 \%$, with no direct correlation between clinical and immunologic responses reported. Recently, a phase II clinical trial was performed in patients with metastatic cervical cancer previously treated with chemotherapy or chemoradiotherapy. Following lymphocyte-depleting chemotherapy, patients received a single infusion of tumor-infiltrating T cells selected for HPV E6 and E7 reactivity. Objective tumor responses were observed in three of the nine patients enrolled: one patient presented with a three-month partial response, and two patients presented with complete responses that were ongoing at 22 and 15 months after treatment, respectively. This proof-of-concept study demonstrated durable, complete regression of metastatic cervical cancer following a single infusion of HPV-specific tumor-infiltrating T cells [52].

Another promising option for therapeutic vaccination is bacterial or viral vector-based vaccines (e.g., sindbis virus, equine encephalitis virus, or adenovirus). Thus far, the efficacy of some viral vector vaccines has been reported in preclinical models, while others have been tested in clinical trials. A recombinant vaccinia virus expressing the E2 protein of HPV-16 or HPV-18 led to complete lesion regression in CIN grade $2 / 3$ patients [53], whereas a vaccine expressing a fusion protein of E6 and E7 caused therapeutic effects in patients with VIN in phase I/II clinical trials [54].

Nevertheless, survival outcomes of patients with HPVassociated cancers treated with the aforementioned vaccine combinations need to be greatly enhanced. 


\section{Axalimogene filolisbac (AXAL or ADXS11-001)}

The bacterial vector most commonly used as an immunotherapeutic vaccine base is Listeria monocytogenes $(L m)$, because of its immunologic advantages. $\mathrm{Lm}$ is a gram-positive intracellular bacterium capable of escaping from the host cell phagosomes into the cytoplasm, thereby infecting host cells. Following infection of host cells, $L m$ has the ability to activate both the innate (neutrophils and macrophages) [55] and adaptive (CD4-positive and CD8-positive T cells) [56] immune responses. In cancer immunotherapy, $L m$ has been successfully used as a delivery vector for tumorspecific antigens.

$L m$-listeriolysin O (LLO) immunotherapies have been reported to present with multiple simultaneous mechanisms of action that contribute to generation of a therapeutic response, enabled by their capacity to efficiently stimulate both innate and adaptive immune responses [57]. Upon administration, Lm-LLO immunotherapies have been shown to infect antigen-presenting cells, thereby initiating the process of antigen cross-presentation. This effect propagates to both arms of the adaptive immune system, leading to generation of activated CD4-positive and CD8-positive T cells. Additionally, $L m$-LLO immunotherapies selectively reduce levels of intratumoral, but not splenic, Tregs and MDSCs, and present the capacity to induce maturation of immune cells to fully differentiated effector cells devoid of protumoral activity. Other advantages of $L m$-LLO immunotherapies are their lack of induction of neutralizing antibodies and their capacity to facilitate chemotaxis of activated immune cells. Interestingly, $L m$-LLO immunotherapies also stimulate robust immune memory responses; correlates of immune memory to $L m$ have been reported to develop just $5 \mathrm{~h}$ after exposure [58]. In various models, Lm-LLO immunotherapies have been shown to also induce therapeutic changes in the ratio of CD8-positive tumorinfiltrating lymphocytes to Tregs [59].

Because of the aforementioned promising results, one such $L m$-LLO immunotherapy was used for development of AXAL, a novel immunotherapeutic agent for treatment of cervical cancer and other HPV-associated diseases. AXAL is based on the live, irreversibly attenuated $\mathrm{Lm}$ fused to the nonhemolytic fragment of LLO, and has been developed to secrete the Lm-LLO-E7 fusion protein targeting HPV-positive tumors [60]. AXAL is also bioengineered to be deficient of virulence-related transcription factors, such as peptide-chain release factor A [61, 62], induces antitumor T-cell immunity, and reduces tumor immune tolerance.

\section{AXAL in clinical trials}

Presently, AXAL is being evaluated in several clinical trials of patients with various HPV-associated tumors (Table 2) [61, 63-71]. Thus far, AXAL has most extensively been evaluated in cervical cancer, with various recently finalized or currently ongoing clinical trials having enrolled patients with cervical cancer at different stages.

\section{AXAL in patients with cervical cancer}

In 2009, Maciag et al. [61] published the first phase I clinical trial of AXAL, in which safety and efficacy were assessed in 15 patients with advanced cervical cancer whose disease presented no improvements following traditional therapies. Women with a history of listeriosis were excluded from this study. Doses of AXAL escalating from $1 \times 10^{9}$ colony-forming units (CFU) to $3.3 \times 10^{9} \mathrm{CFU}$ and then $1 \times 10^{10} \mathrm{CFU}$ were administered to groups of five patients every 21 days for a total of two intravenous doses. Patients also received prophylactic antibiotics. Dose-limiting toxicity was achieved at the highest dose of $1 \times 10^{10} \mathrm{CFU}$, with three of five patients developing hemodynamic instability and subsequently treated with medical interventions. All 15 patients reported at least one $\mathrm{AE}$, as classified by the Common Terminology Criteria for Adverse Events version 3.0 [72]. The most common AEs reported by more than $50 \%$ of patients during the study were pyrexia (100\%), vomiting $(60 \%)$, musculoskeletal pain (57\%), and chills, headache, and anemia (53\%). Blood, urine, and feces analyses revealed the transient presence of $L m$-LLO-E7 in only one patient receiving $1 \times 10^{9} \mathrm{CFU}$. Within the follow-up period of the study, two deaths were recorded. One death was caused by disease progression, while the second occurred in the setting of renal failure, followed by metabolic acidosis and cardiac arrest. Both were deemed unrelated to AXAL [61]. Overall, the results of this study showed an acceptable safety profile for AXAL at the dose of $1 \times 10^{9} \mathrm{CFU}$.

Considering the acceptable safety profile of AXAL observed in the study of Maciag et al., a single-arm, two-stage, phase II clinical trial is being conducted in patients with squamous or nonsquamous persistent, recurrent, metastatic cervical cancer that has progressed after systemic chemotherapy. In a Gynecologic Oncology Group (GOG) study, a total of 67 patients was estimated for enrolment at study initiation, with a target of 27 patients enrolled in the first study stage [63] (Table 2) [61, 63-71]. Eligible patients are 18 years of age or older, have a GOG performance status of 0 or 1 , have measurable disease (Response Evaluation Criteria in Solid Tumors [RECIST] version 1.1), and have received one or more prior lines of systemic-dose chemotherapy (bevacizumab permitted) for squamous or non-squamous persistent or recurrent metastatic cervical cancer not amenable to curative therapy. In this study, AXAL safety and tolerability, as well as 12-month OS rates, were evaluated following administration of three doses of AXAL at 


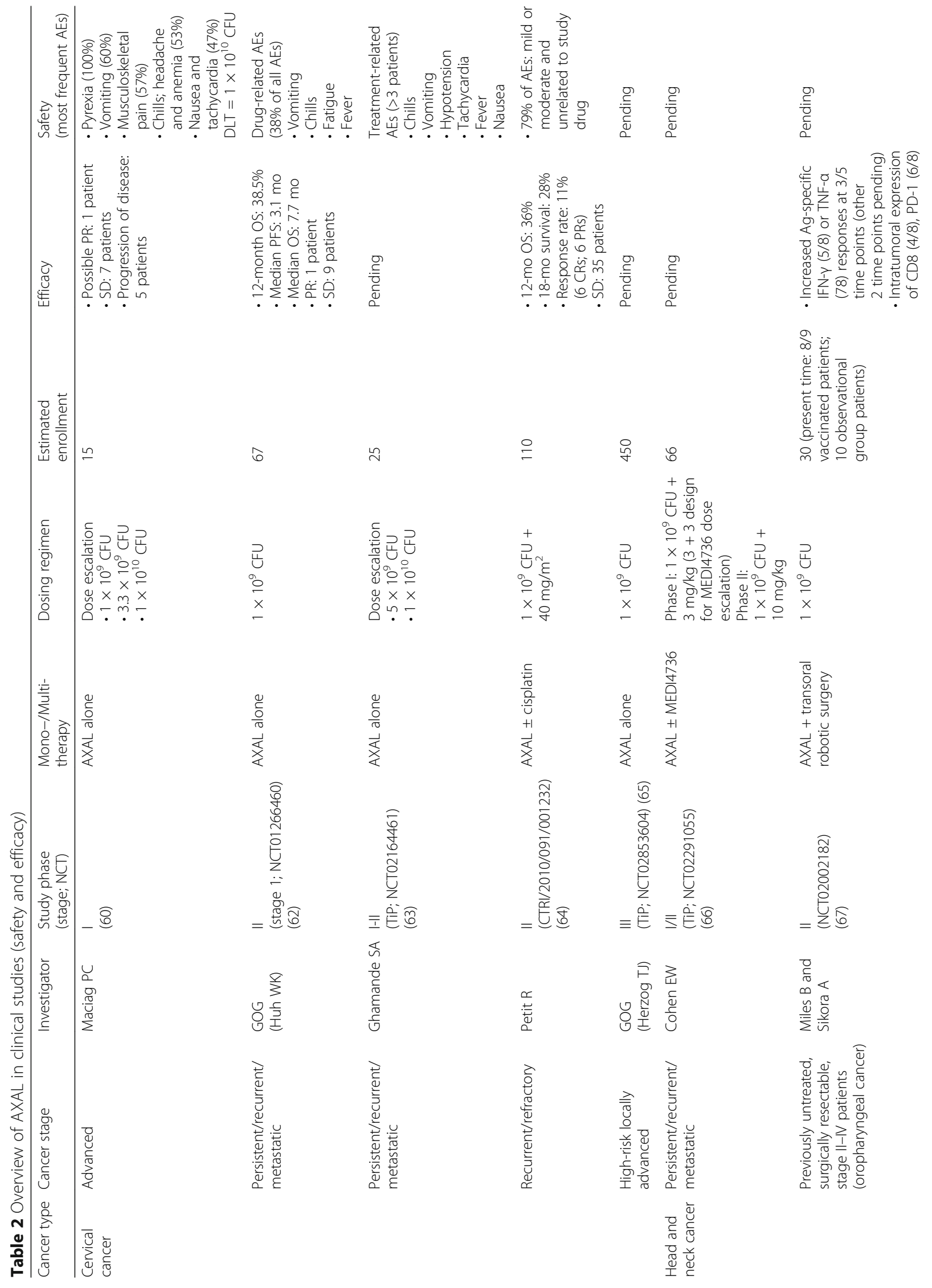




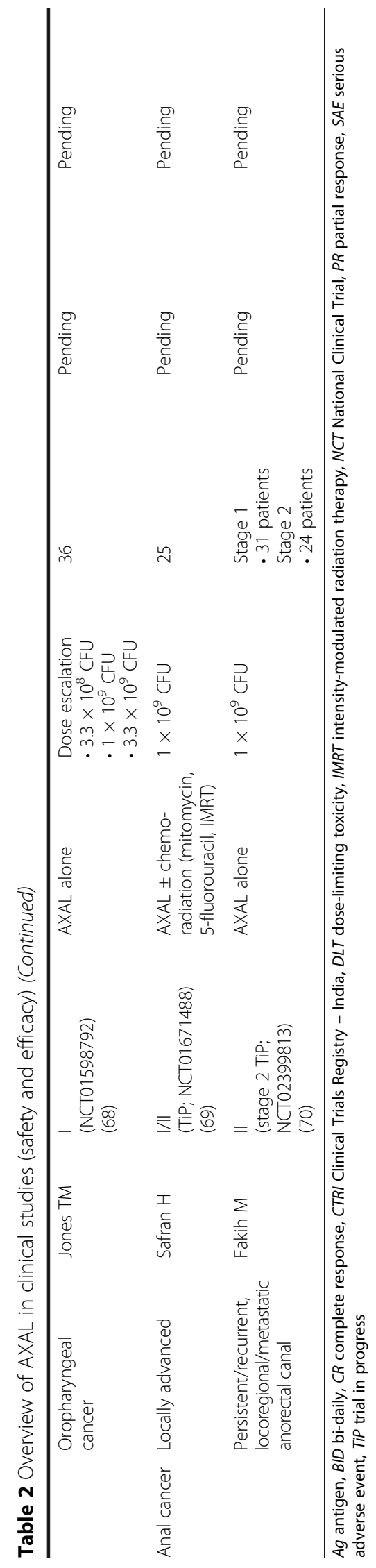


$1 \times 10^{9}$ CFU every 28 days. Secondary endpoints were PFS, OS, and objective response (OR). To prevent development of the most common AEs reported in the study by Maciag et al., nonsteroidal anti-inflammatory agents were administered prophylactically. In total, 26 of the 29 patients who were enrolled in stage 1 received treatment. Safety analyses indicated that all treated patients experienced at least one AE: $91 \%$ were grade 1-2, and 38\% were drug related, with nausea, vomiting, chills, fatigue, and fever the most common. The $38.5 \%$ (10 patients) 12-month OS rate observed in stage 1 of the study suggests that AXAL is an active agent with a net survival benefit for patients with squamous or nonsquamous persistent or recurrent metastatic cervical cancer. Median PFS was 3.1 months and median OS was 7.7 months. Preliminary evaluation of OR showed that one patient presented with unconfirmed partial response and nine patients presented with stable disease. Post-hoc efficacy analysis of the 18 patients who received all three per-protocol doses of AXAL showed a median OS longer than 1 year, and a 12-months OS rate of 55.6\%, thus confirming the survival benefit offered by AXAL.

Another phase I, open-label, dose-escalation clinical trial being performed in patients with persistent, recurrent, or metastatic cervical squamous carcinoma or adenocarcinoma aims to evaluate the safety and tolerability of higher doses of AXAL, as well as tumor response, PFS, and correlative immunologic parameters [64] (Table 2) [61, 63-71]. Patients enrolled in this study had measurable disease (RECIST version 1.1) with documented disease progression on or intolerance to prior therapy, and had an Eastern Cooperative Oncology Group (ECOG) performance status of 0 or 1 . Overall, 10 of 25 patients were enrolled, and nine of 10 patients were treated with AXAL every 3 weeks during a 12week treatment cycle (six patients received $5 \times 10^{9} \mathrm{CFU}$ and three patients received $1 \times 10^{10} \mathrm{CFU}$ ). The primary endpoint of this study was AXAL safety and tolerability, with the recommended phase II dose (RP2D) selected based on a dose-limiting toxicity rate lower than 33\%. Secondary objectives included evaluation of tumor response and PFS. All treated patients experienced at minimum one $\mathrm{AE}$, of which $75 \%$ were TRAEs (eight of nine patients): $99 \%$ were grade $1-2$, and the most common TRAEs occurring in three or more patients were chills, vomiting, hypotension, tachycardia, fever, and nausea. Only one grade 3 (hypotension) and no grade 4-5 TRAEs were reported. Analysis of tumor response and PFS, as well as correlative immunologic studies, is ongoing to assess if treatment intensity has an impact on the antitumor activity of AXAL. Lastly, another phase III clinical trial of AXAL (AIM2CERV) administered as adjuvant immunotherapy in patients with high-risk, locally advanced cervical cancer following chemoradiation was opened for recruitment in September 2016 [65] (Table 2) [61, 63-71].

Given the observed safety and efficacy of AXAL when administered alone in patients with cervical cancer, combinatorial therapies containing AXAL were also assessed in clinical trials. The efficacy and safety of AXAL, administered with or without cisplatin, was evaluated in a phase II trial performed in India that enrolled 110 patients with recurrent or progressive invasive cervical cancer unresponsive to primary therapy [65] (Table 2) [61, 63-71]. Eligible patients were 18 years of age or older, had documented recurrent or progressing invasive cervical cancer, presented with measurable disease with at least one target lesion, and had ECOG performance status 2 or lower. Patients were randomized to AXAL alone (one cycle of three doses at $1 \times 10^{9} \mathrm{CFU}$ administered every 4 weeks) or AXAL plus cisplatin (one preliminary AXAL dose followed by five weekly cisplatin treatments at the dose of $40 \mathrm{mg} / \mathrm{m}^{2}$, followed by one AXAL cycle). The primary endpoint was OS; secondary endpoints were OR rates, PFS, and safety. Among the 109 patients who received treatment, AXAL was well tolerated; $79 \%$ of AEs were mild or moderate and unrelated to study drug. OS was found to be similar between the two treatment arms (median OS AXAL: 8.40 months; AXAL with cisplatin: 8.77 months). Furthermore, $22 \%$ of the treated patients alive at more than 18 months following AXAL therapy were deemed long-term survivors. No significant differences between the OR rates, disease control rates, duration of response, or PFS were observed between the two treatment groups.

\section{AXAL in patients with head and neck cancer}

In addition to the clinical trials performed in patients with cervical cancer, AXAL is also being investigated in other types of HPV-positive cancers, such as head and neck and anorectal cancer. Three phase I/II clinical trials in patients with head and neck cancer and two phase I/ II clinical trials in patients with anorectal cancer are currently ongoing. The phase I/II randomized two-stage study by Cohen et al. is being performed in patients with recurrent, HPV-positive squamous cell carcinoma of the head and neck or cervix [67] (Table 2) [61, 63-71]. In phase I of this study, safety and efficacy of $1 \times 10^{9} \mathrm{CFU}$ of AXAL administered every 4 weeks in combination with the PD-1 inhibitor durvalumab (MEDI-4736), used at escalating doses (dose level $1: 3 \mathrm{mg} / \mathrm{kg}$; dose level 2: $10 \mathrm{mg} / \mathrm{kg}$ ) administered every 2 weeks, will be assessed, and the RP2D of the combination therapy will be determined in up to 18 patients. In phase II of the study, 48 patients will be randomized to receive AXAL $\left(1 \times 10^{9} \mathrm{CFU}\right)$, durvalumab, or both, at the preestablished RP2D. The study allows patients to receive 
treatment for up to 1 year or, alternatively, discontinue treatment due to disease progression or unacceptable toxicity, and is currently ongoing.

Another clinical study of AXAL in patients with previously untreated, surgically resectable, stage II-IV oropharyngeal cancer is the "window of opportunity" phase II, two-stage trial that was initiated in 2014 [68] (Table 2) [61, 63-71]. Patients received AXAL at $1 \times 10^{9} \mathrm{CFU}$ (two doses over 5 weeks, on the first and fifteenth days of treatment, respectively), prior to standard-of-care transoral robotic surgery. Of the eight of nine enrolled patients who completed study treatment, five patients presented with increased peripheral blood antigen-specific interferon gamma $($ IFN- $\gamma)$ or tumor necrosis factor alpha (TNF- $\alpha$ ) responses before treatment, on the day of surgery, and 5 weeks postsurgery. Additionally, intratumoral increases in posttreatment expression of CD8-positive $\mathrm{T}$ cells and PD-1 were recorded in four of eight and six of eight patients, respectively. These results are promising, as they indicate that effects of AXAL are not limited to the generation of robust antitumoral immune responses, but are also extended to the tumor microenvironment. Another phase I clinical trial evaluating the safety of escalating AXAL doses (from $3.3 \times 10^{8}$ to $\left.3.3 \times 10^{9} \mathrm{CFU}\right)$ in oropharyngeal cancer is also in progress [63] (Table 2) [61, 63-71].

\section{AXAL in patients with anal cancer}

In view of the reported efficacy of AXAL in cervical cancer and available immune response data in head and neck cancers, clinical trials aiming to evaluate this immunotherapeutic compound in HPV-positive anal cancers have recently been initiated. The phase I/II study by Safran et al. aims to assess safety and efficacy of AXAL when combined with intensity-modulated radiation therapy, mitomycin, and 5-fluorouracil for treatment of patients with anal cancer [70] (Table 2) [61, 63-71]. The phase II, two-stage study by Fakih et al. aims to assess the efficacy and safety of AXAL monotherapy (administered intravenously at a dose of $1 \times 10^{9} \mathrm{CFU}$ every 3 weeks during nine-week treatment cycles) in patients with persistent, recurrent, locoregional, or metastatic squamous cell cancer of the anus. [71] (Table 2) [10, 63-71]. Both of these trials are ongoing, and results are expected to be made available in 2017.

\section{Advantages of treatment with AXAL and future perspectives}

AXAL seems to embody the main characteristics of a successful HPV therapeutic vaccine for patients with various $\mathrm{HPV}$-associated cancers, both in terms of efficacy (i.e., capacity to engage both innate and adaptive immunity by promoting inflammation and inducing high numbers of antigen-specific cytotoxic $\mathrm{T}$ lymphocytes) and safety (i.e., reported tolerability). Additional benefits include the ability of AXAL to dampen intratumoral immune tolerance by reducing numbers and functionality of Tregs and MDSCs, as well as reducing secretion of immunosuppressive cytokines (e.g., interleukin-10 and transforming growth factor beta), both features of an immune response that would ordinarily contribute to a protumor microenvironment. Another advantage of AXAL immunotherapy is exemplified by its delivery vector, $L m$, an attenuated bacterial vector characterized by lack of virulence yet retaining its adjuvant properties. Moreover, given the conserved nature of the E7 early gene in HPV, AXAL is expected to be effective irrespective of the HPV serotype associated with individual cases, which is particularly important in heterogeneous patient populations such as those with head and neck cancer. A further advantageous aspect of AXAL therapy is its potential to provide longterm immune modulation, an aspect of treatment that is currently lacking. In the case of HPVassociated oropharyngeal squamous cell carcinomas, which are known to involve longer survival and later recurrence compared to other head and neck cancers [72], the use of AXAL to bolster the antitumor response in conjunction with disease surveillance is an exciting yet currently unexplored option for preventing late recurrence in this setting. Potentially, treatment with AXAL could have an immediate impact improving patients' perception of care, which is often negatively affected by delays in scheduling surgery or radiotherapy. The effective and safe administration of AXAL in the weeks prior to definitive treatment has been indicated to be plausible in the window of opportunity study [68]. Another study that may have significant bearing on future strategies for AXAL use is AIM2CERV, the randomized phase III study of AXAL use following chemoradiation in patients with high-risk, locally advanced cervical cancer [66] (Fig. 2). In this setting, there is a clear unmet need, as patients have a $50 \%$ probability of disease recurrence or death following cisplatin-based chemoradiation plus brachytherapy. AIM2CERV will evaluate disease-free and OS as its endpoints; study enrollment is currently ongoing. Based on the studies described above and the windows of opportunity naturally occurring in different therapeutic scenarios, future directions of treatment with AXAL will most likely include administration in oropharyngeal squamous cell carcinomas and high-risk locally advanced cervical cancer, in combination with standard treatment options or alone. 


\section{Schema - ADXS AIM2CERV Study}

\section{Randomization 1:2 Between Reference and Treatment Groups}

\begin{tabular}{|l|}
\hline High-risk, locally advanced \\
cervical cancer \\
- FIGO stage IB2-II with positive \\
pelvic nodes \\
- FIGO stage III-IV \\
- Any FIGO stage with \\
para-aortic nodes \\
Total sites: 150 sites in 20 \\
countries \\
- GOG is supporting AIM2CERV \\
by acting as a Site Management \\
Organization \\
Trial timeline (predicted) \\
- First patient enrollment: $3 Q 16$ \\
- Last patient enrollment: $2 \mathrm{Q} 18$ \\
Final data readout: $3 \mathrm{Q} 19$ \\
\end{tabular}

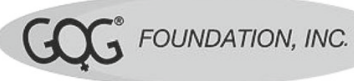

Cisplatin (at least 4 weeks exposure) and Radiation (minimum 40-Gy external beam radiation therapy)

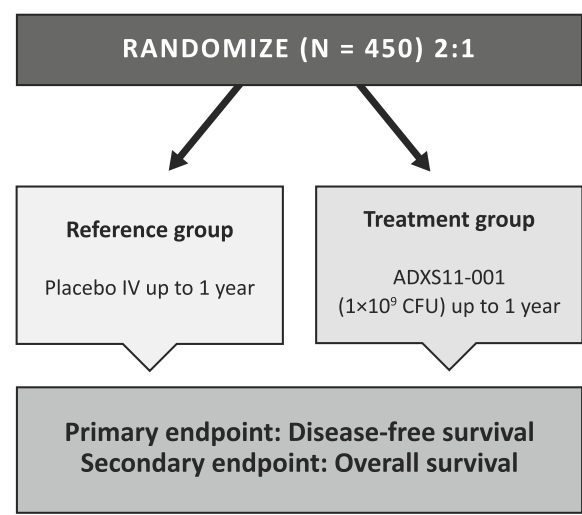

Participating countries:

Argentina, Brazil, Canada, Chile, Hong Kong, Ireland, Korea, Malaysia, Mexico, Netherlands, Poland, Romania, Russia, Serbia, Spain, Taiwan, UK, Ukraine, US

Fig. 2 AXAL planned phase III study. AXAL: axalimogene filolisbac; FIGO: International Federation of Gynecology and Obstetrics; GOG: Gynecologic Oncology Group; Q: quarter; UK: United Kingdom; US: United States

\section{Conclusions}

Considering the rising incidence of various HPVassociated cancers within the last years [73, 74], as well as HPV infection being more recently established as the principal cause of increased incidence in head and neck cancers [74], novel therapeutic options for HPVassociated cancers are stringently necessary. Novel therapeutic options for HPV-associated cancers include the use of vaccines based on DNA, peptides, or viral vectors. Delivery of HPV antigens using viral vectors, such as in the case of AXAL, has several advantages: in contrast to peptide immunization, CTL epitopes can be processed/presented naturally and delivered more effectively to target cells; in contrast to DNA immunization, the efficiency of introducing heterologous genes in target cells can be enhanced. While the exact costs for treatment with AXAL are not yet completely elucidated, given the long-term beneficial effects reported upon its administration to patients with HPV-positive tumors, it can be hypothesized that AXAL treatment will be cost-effective. Furthermore, considering the relatively straightforward method of AXAL production, the costs of a full course of AXAL are hypothesized to be far inferior to those of Sipuleucel-T treatment, for which a full course rises to $\$ 98,780$ [75]. A more specific cost-estimate of the treatment will hopefully be possible in the near future, especially since treatment with AXAL is expected to be available within the next 5 years or earlier. In this regard, patients with high-risk locally advanced cervical cancer represent the ideal target patients for treatment with AXAL, also based on the promising results obtained so far in this patient population.

Although there is evidence in the literature demonstrating a growing incidence of decreased vaccine acceptance and hesitancy of usage [76], the various ongoing clinical trials with AXAL indicate its potential for widespread use across various types of HPV-associated cancers, thus placing AXAL among the promising immunotherapeutic tools of the future.

\section{Abbreviations}

Ag: Antigen; AIN: Anal intraepithelial neoplasia; AXAL: Axalimogene filolisbac; BID: bi-daily; CD: Cluster of differentiation; CFU: Colony-forming unit; CIN: Cervical intraepithelial neoplasia; CR: Complete response; CTRI: Clinical Trials Registry - India; DLT: Dose-limiting toxicity; DNA: Deoxyribonucleic acid; ECOG: Eastern Cooperative Oncology Group; FIGO: Federation of Gynecology and Obstetrics; GOG: Gynecologic Oncology Group; HPV: Human papillomavirus; IFN-ү: Interferon gamma; IMRT: Intensity-modulated radiation therapy; LLO: Listeriolysin O; Lm: Listeria monocytogenes; MDSCs: Myeloidderived suppressor cells; NCT: National Clinical Trial; OR: Objective response; OS: Overall survival; PD-1: Programmed death protein 1; PFS: Progression-free survival; PR: Partial response; Q: Quarter; RECIST: Response Evaluation Criteria 
In Solid Tumors; RP2D: Recommended phase II dose; TiP: Trial in progress; TNF-a: Tumor necrosis factor alpha; TRAEs: Treatment-related adverse events; Tregs: Regulatory T cells; UK: United Kingdom; US: United States; VIN: Vulvar intraepithelial neoplasia

\section{Acknowledgements}

The authors would like to thank Oana Draghiciu, PhD, from TRM Oncology, for medical writing assistance, funded by Advaxis, Inc. The authors are fully responsible for all content and editorial decisions for this review.

\section{Funding}

Medical writing assistance was funded by Advaxis, Inc.

\section{Availability of data and materials}

Data sharing is not applicable to this article as no datasets were generated or analyzed during the current study.

\section{Authors' contributions}

$B M, H P S$, and BJM were integral in the writing, review, and revision of the manuscript. All authors read and approved the final manuscript.

\section{Ethics approval and consent to participate}

All patients participating in the clinical trials reported in this review have signed a written informed consent for participation in the trials. The studies were approved by Independent Review Boards at each study site.

\section{Consent for publication}

Not applicable.

\section{Competing interests}

$\mathrm{BM}$ has received research funding for the Window of Opportunity clinical trial (NCT02002182) from Advaxis Inc. BJM discloses that the St. Joseph's Hospital institution has received research grants from Amgen, Lilly, Genentech, Janssen/Johnson \& Johnson, Array, TESARO, and Morphotek. BJM has received honoraria for speaker bureaus from Roche/Genentech, AstraZeneca, Myriad, and Janssen/Johnson \& Johnson; and has been a consultant for Roche/Genentech, Merck, TESARO, AstraZeneca, Gradalis, Advaxis, Amgen, Pfizer, Bayer, Insys, Mateon (formerly OXiGENE), PPD, and Clovis. HPS has nothing to disclose.

\section{Publisher's Note}

Springer Nature remains neutral with regard to jurisdictional claims in published maps and institutional affiliations.

\section{Author details}

'Department of Otolaryngology, Icahn School of Medicine at Mount Sinai, New York, NY, USA. ${ }^{2}$ Brown University Oncology Research Group, Providence, RI, USA. ${ }^{3}$ Division of Gynecologic Oncology, Arizona Oncology (US Oncology Network), University of Arizona College of Medicine, Creighton University School of Medicine at St. Joseph's Hospital, 2222 E. Highland Ave, Suite 400 Phoenix, AZ 85016, USA.

Received: 7 February 2017 Accepted: 3 July 2017

Published online: 14 July 2017

\section{References}

1. Forman D, de Martel C, Lacey CJ, Soerjomataram I, Lortet-Tieulent J, Bruni $L$, et al. Global burden of human papillomavirus and related diseases. Vaccine. 2012;30(suppl 5):F12-23.

2. de Martel C, Ferlay J, Franceschi S, Vignat J, Bray F, Forman D, et al. Global burden of cancers attributable to infections in 2008: a review and synthetic analysis. Lancet Oncol. 2012;13(6):607-15.

3. Plummer M, de Martel C, Vignat J, Ferlay J, Bray F, Franceschi S. Global burden of cancers attributable to infections in 2012: a synthetic analysis. Lancet Glob Health. 2016;4(9):e609-16.

4. Scheurer ME, Tortolero-Luna G, Adler-Storthz K. Human papillomavirus infection: biology, epidemiology, and prevention. Int J Gynecol Cancer. 2005;15(5):727-46.

5. Moody CA, Laimins LA. Human papillomavirus oncoproteins: pathways to transformation. Nat Rev Cancer. 2010;10(8):550-60.
6. Bodily J, Laimins LA. Persistence of human papillomavirus infection: keys to malignant progression. Trends Microbiol. 2011;19(1):33-9.

7. Grabowska AK, Riemer AB. The invisible enemy - how human papillomaviruses avoid recognition and clearance by the host immune system. Open Virol J. 2012;6:249-56.

8. Howie HL, Katzenellenbogen RA, Galloway DA. Papillomavirus E6 proteins. Virology. 2009;384(2):324-34.

9. Wise-Draper TM, Wells SI. Papillomavirus E6 and E7 proteins and their cellular targets. Front Biosci. 2008;13:1003-17.

10. Münger K, Basile JR, Duensing S, Eichten A, Gonzalez SL, Grace M, et al. Biological activities and molecular targets of the human papillomavirus E7 oncoprotein. Oncogene. 2001;20(54):7888-98.

11. Bernard HU, Burk RD, Chen Z, van Doorslaer $K$, Zur Hausen $H$, de Villiers EM. Classification of papillomaviruses (PVs) based on 189 PV types and proposal of taxonomic amendments. Virology. 2010;401(1):70-9.

12. Ling M, Kanayama M, Roden R, Wu TC. Preventive and therapeutic vaccines for human papillomavirus-associated cervical cancers. J Biomed Sci. 2000; 7(5):341-56

13. Muñoz N, Bosch FX, de Sanjosé $S$, Herrero R, Castellsaqué $X$, Shah KV, et al. Epidemiologic classification of human papillomavirus types associated with cervical cancer. N Engl J Med. 2003;348(6):518-27.

14. Schiffman M, Clifford G, Buonaguro FM. Classification of weakly carcinogenic human papillomavirus types: addressing the limits of epidemiology at the borderline. Infect Agent Cancer. 2009;4:8.

15. Guan P, Howell-Jones R, Li N, Bruni L, de Sanjosé S, Franceschi S, et al. Human papillomavirus types in 115,789 HPV-positive women: a metaanalysis from cervical infection to cancer. Int J Cancer. 2012;131(10):2349-59.

16. Chaturvedi AK. Beyond cervical cancer: burden of other HPV-related cancers among men and women. J Adolesc Health. 2010;46(4 suppl):S20-6.

17. FUTURE II Study Group. Quadrivalent vaccine against human papillomavirus to prevent high-grade cervical lesions. N Engl J Med. 2007;356(19):1915-27.

18. Centers for Disease Control and Prevention (CDC). FDA licensure of bivalent human papillomavirus vaccine (HPV2, Cervarix) for use in females and updated HPV vaccination recommendations from the Advisory Committee on Immunization Practices (ACIP). MMWR Morb Mortal Wkly Rep. 2010; 59(20):626-9.

19. Petrosky E, Bocchini JA Jr, Hariri S, Chesson H, Curtis CR, Saraiya M, et al. Use of 9-valent human papillomavirus (HPV) vaccine: updated HPV vaccination recommendations of the advisory committee on immunization practices. MMWR Morb Mortal Wkly Rep. 2015;64(11):300-4.

20. Stern PL, van der Burg SH, Hampson IN, Broker TR, Fiander A, Lacey CJ, et al. Therapy of human papillomavirus-related disease. Vaccine. 2012;30(suppl 5): F71-82.

21. Milinovic D, Kalafatic D, Babic D, Oreskovic LB, Grsic HL, Oreskovic S. Minimally invasive therapy of cervical intraepithelial neoplasia for fertility preservation. Pathol Oncol Res. 2009;15(3):521-5.

22. The Cancer Genome Atlas Research Network. Integrated genomic and molecular characterization of cervical cancer. Nature. 2017;541:169-75.

23. Rose PG. Combined-modality therapy of locally advanced cervical cancer. J Clin Oncol. 2003;21(10 suppl):211s-7s

24. Moore DH, Blessing JA, McQuellon RP, Thaler HT, Cella D, Benda J, et al. Phase III study of cisplatin with or without paclitaxel in stage IVB, recurrent, or persistent squamous cell carcinoma of the cervix: a gynecologic oncology group study. J Clin Oncol. 2004;22(15):3113-9.

25. Monk BJ, Sill MW, McMeekin DS, Cohn DE, Ramondetta LM, Boardman CH, et al. Phase III trial of four cisplatin-containing doublet combinations in stage IVB, recurrent, or persistent cervical carcinoma: a Gynecologic Oncology Group study. J Clin Oncol. 2009;27(28):4649-55.

26. Tewari KS, Sill MW, Long HJ 3rd, Penson RT, Huang H, Ramondetta LM, et al. Improved survival with bevacizumab in advanced cervical cancer. N Engl J Med. 2014:370(8):734-43.

27. Gill BS, Bernard ME, Lin JF, Balasubramani GK, Rajagopalan MS, Sukumvanich $P$, et al. Impact of adjuvant chemotherapy with radiation for node-positive vulvar cancer: A National Cancer Data Base (NCDB) analysis. Gynecol Oncol. 2015;137(3):365-72.

28. National Comprehensive Cancer Network. NCCN Clinical Practice Guidelines in Oncology (NCCN Guidelines ${ }^{\circledast}$ ). Vulvar cancer (squamous cell carcinoma). Version 1.2017. 2016. https://www.nccn.org/professionals/physician_gls/f_ guidelines.asp. Accessed 30 Nov 2016

29. Eifel PJ, Berek JS, Markman MA. Cancer of the cervix, vagina, and vulva. In: DeVita Jr VT, Lawrence TS, Rosenberg SA, editors. DeVita, Hellman, and 
Rosenberg's Cancer: Principles and Practice of Oncology. 9th ed. Philadelphia, Pa: Lippincott Williams \& Wilkins; 2011. p. 1311-44.

30. Samant R, Lau B, Choan E, Le T, Tam T. Primary vaginal cancer treated with concurrent chemoradiation using Cis-platinum. Int J Radiat Oncol Biol Phys. 2007;69(3):746-50.

31. Olthof NC, Straetmans JM, Snoeck R, Ramaekers FC, Kremer B, Speel EJ. Next-generation treatment strategies for human papillomavirus-related head and neck squamous cell carcinoma: where do we go? Rev Med Virol. 2012;22(2):88-105.

32. Chaturvedi AK, Engels EA, Pfeiffer RM, Hernandez BY, Xiao W, Kim E, et al. Human papillomavirus and rising oropharyngeal cancer incidence in the United States. J Clin Oncol. 2011;29(32):4294-301.

33. Varier I, Keeley BR, Krupar R, Patsias A, Dong J, Gupta N, et al. Clinical characteristics and outcomes of oropharyngeal carcinoma related to high-risk non-human papillomavirus16 viral subtypes. Head Neck. 2016; 38(9):1330-7.

34. Truong Lam M, O'Sullivan B, Gullane P, Huang SH. Challenges in establishing the diagnosis of human papillomavirus-related oropharyngeal carcinoma. Laryngoscope. 2016;126(10):2270-5.

35. Cracchiolo JR, Baxi SS, Morris LG, Ganly I, Patel SG, Cohen MA, et al. Increase in primary surgical treatment of $\mathrm{T} 1$ and $\mathrm{T} 2$ oropharyngeal squamous cell carcinoma and rates of adverse pathologic features: National Cancer Data Base. Cancer. 2016;122(10):1523-32.

36. Ferris RL, Blumenschein G Jr, Fayette J, Guigay J, Colevas AD, Licitra L, et al. Nivolumab for recurrent squamous-cell carcinoma of the head and neck. N Engl J Med. 2016;375(19):1856-67.

37. Goldstone SE, Kawalek AZ, Huyett JW. Infrared coagulator: a useful tool for treating anal squamous intraepithelial lesions. Dis Colon rectum. 2005;48(5):1042-54

38. Leon O, Guren MG, Radu C, Gunnlaugsson A, Johnsson A. Phase I study of cetuximab in combination with 5 -fluorouracil, mitomycin $\mathrm{C}$ and radiotherapy in patients with locally advanced anal cancer. Eur J Cancer. 2015;51(17):2740-6.

39. National Comprehensive Cancer Network. NCCN Clinical Practice Guidelines in Oncology (NCCN Guidelines ${ }^{\circledast}$ ). Anal carcinoma. Version 1.2017. 2016. https://www.nccn.org/professionals/physician_gls/f guidelines.asp. Accessed 30 Nov 2016.

40. Di Lorenzo G, Buonerba C, Ferro M, Calderoni G, Bozza G, Federico P, et al. The epidermal growth factor receptors as biological targets in penile cancer. Expert Opin Biol Ther. 2015;15(4):473-6.

41. National Comprehensive Cancer Network. NCCN Clinical Practice Guidelines in Oncology (NCCN Guidelines ${ }^{\circledast}$ ). Penile cancer. Version 2. 2016. 2016. https://www.nccn.org/professionals/physician_gls/f_guidelines.asp. Accessed 30 Nov 2016.

42. van Poelgeest MI, van Seters M, van Beurden M, Kwappenberg KM, HeijmansAntonissen C, Drijfhout JW, et al. Detection of human papillomavirus (HPV) 16specific CD4+ T-cell immunity in patients with persistent HPV16-induced vulvar intraepithelial neoplasia in relation to clinical impact of imiquimod treatment. Clin Cancer Res. 2005;11(14):5273-80

43. Kobayashi A, Weinberg V, Darragh T, Smith-McCune K. Evolving immunosuppressive microenvironment during human cervical carcinogenesis. Mucosal Immunol. 2008;1(5):412-20.

44. Kenter GG, Welters MJ, Valentijn AR, Lowik MJ, Berends-van der Meer DM, Vloon AP, et al. Phase I immunotherapeutic trial with long peptides spanning the E6 and E7 sequences of high-risk human papillomavirus 16 in end-stage cervical cancer patients shows low toxicity and robust immunogenicity. Clin Cancer Res. 2008;14(1):169-77.

45. Palefsky JM, Berry JM, Jay N, Krogstad M, Da Costa M, Darragh TM, et al. A trial of SGN-00101 (HspE7) to treat high-grade anal intraepithelial neoplasia in HIV-positive individuals. AIDS. 2006;20(8):1151-5.

46. Frazer $\mathbb{H}$, Quinn $\mathrm{M}$, Nicklin $\mathrm{JL}$, Tan J, Perrin $\mathrm{LC}, \mathrm{Ng}$ P, et al. Phase 1 study of HPV16-specific immunotherapy with E6E7 fusion protein and ISCOMATRIX adjuvant in women with cervical intraepithelial neoplasia. Vaccine. 2004; 23(2):172-81.

47. Roman LD, Wilczynski S, Muderspach LI, Burnett AF, O'Meara A, Brinkman $J$ A, et al. A phase II study of Hsp-7 (SGN-00101) in women with high-grade cervical intraepithelial neoplasia. Gynecol Oncol. 2007;106(3):558-66.

48. Welters MJ, Kenter GG, Piersma SJ, Vloon AP, Löwik MJ, Berends-van der Meer DM, et al. Induction of tumor-specific CD4+ and CD8+ T-cell immunity in cervical cancer patients by a human papillomavirus type 16 E6 and E7 long peptides vaccine. Clin Cancer Res. 2008;14(1):178-87.
49. Trimble CL, Peng S, Kos F, Gravitt P, Viscidi R, Sugar E, et al. A phase I trial of a human papillomavirus DNA vaccine for HPV16+ cervical intraepithelial neoplasia 2/3. Clin Cancer Res. 2009;15(1):361-7.

50. Santin AD, Bellone S, Palmieri M, Zanolini A, Ravaggi A, Siegel ER, et al. Human papillomavirus type 16 and 18 E7-pulsed dendritic cell vaccination of stage IB or IIA cervical cancer patients: a phase I escalating-dose trial. J Virol. 2008;82(4):1968-79.

51. Ferrara A, Nonn M, Sehr P, Schreckenberger C, Pawlita M, Dürst M, et al. Dendritic cell-based tumor vaccine for cervical cancer II: results of a clinical pilot study in 15 individual patients. J Cancer Res Clin Oncol. 2003;129(9):521-30.

52. Stevanović S, Draper LM, Langhan MM, Campbell TE, Kwong ML, Wunderlich JR, et al. Complete regression of metastatic cervical cancer after treatment with human papillomavirus-targeted tumor-infiltrating T cells. J Clin Oncol. 2015;33(14):1543-50.

53. García-Hernández E, González-Sánchez JL, Andrade-Manzano A, Contreras ML, Padilla S, Guzmán CC, et al. Regression of papilloma high-grade lesions (CIN 2 and CIN 3) is stimulated by therapeutic vaccination with MVA E2 recombinant vaccine. Cancer Gene Ther. 2006;13(6):592-7.

54. Davidson EJ, Faulkner RL, Sehr P, Pawlita M, Smyth LJ, Burt DJ, et al. Effect of TA-CIN (HPV 16 L2E6E7) booster immunisation in vulval intraepithelial neoplasia patients previously vaccinated with TA-HPV (vaccinia virus encoding HPV 16/18 E6E7). Vaccine. 2004;22(21-22):2722-9.

55. Zenewicz LA, Shen H. Innate and adaptive immune responses to Listeria monocytogenes: a short overview. Microbes Infect. 2007;9(10):1208-15.

56. Pan ZK, Ikonomidis G, Lazenby A, Pardoll D, Paterson Y. A recombinant Listeria monocytogenes vaccine expressing a model tumour antigen protects mice against lethal tumour cell challenge and causes regression of established tumours. Nat Med. 1995;1(5):471-7.

57. Rothman J, Wallecha A, Maciag PC, Rivera S, Shahabi V, Paterson Y. The use of living Listeria monocytogenes as an active immunotherapy for the treatment of cancer. In: Fialho AM, Chakrabarty A, editors. Emerging cancer therapy: microbial approaches and biotechnological tools. New York: John Wiley \& Sons, Inc.; 2010. p. 13-48.

58. Campisi L, Soudja SM, Cazareth J, Bassand D, Lazzari A, Brau F, et al. Splenic $\mathrm{CD} 8 \mathrm{a}^{+}$dendritic cells undergo rapid programming by cytosolic bacteria and inflammation to induce protective $\mathrm{CD}^{+} \mathrm{T}$-cell memory. Eur J Immunol. 2011;41(6):1594-605.

59. Shahabi V, Reyes-Reyes M, Wallecha A, Rivera S, Paterson Y, Maciag P. Development of a Listeria monocytogenes based vaccine against prostate cancer. Cancer Immunol Immunother. 2008;57(9):1301-13.

60. Wallecha A, French C, Petit R, Singh R, Amin A, Rothman J. Lm-LLO-based immunotherapies and HPV-associated disease. J Oncol. 2012;2012:542851.

61. Maciag $P C$, Radulovic $S$, Rothman J. The first clinical use of a live-attenuated Listeria monocytogenes vaccine: a Phase I safety study of Lm-LLO-E7 in patients with advanced carcinoma of the cervix. Vaccine. 2009;27(30):3975-83.

62. de las Heras A, Cain RJ, Bielecka MK, Vázquez-Boland JA. Regulation of Listeria virulence: PrfA master and commander. Curr Opin Microbiol. 2011;14(2):118-27.

63. Huh WK, Brady WE, Moore KN, Lankes HA, Monk BJ, Aghajanian C, et al. A phase 2 study of live-attenuated Listeria monocytogenes cancer immunotherapy (ADXS11-001) in the treatment of persistent or recurrent cancer of the cervix (GOG-0265). J Clin Oncol. 2014;32(suppl): abstract TPS5617.

64. Ghamande SA, Dobbins R, Marshall L, Wheatley D, Price C, Mauro DJ, et al. Phase I study evaluating high dose ADXS11-001 treatment in women with carcinoma of the cervix. J Clin Oncol. 2015;33(suppl): abstract TPS3096.

65. Petit RG, Mehta A, Jain M, Gupta S, Nagarkar R, Kumar V, et al. ADXS11-001 immunotherapy targeting HPV-E7: final results from a Phase II study in Indian women with recurrent cervical cancer. J Immunother Cancer. 2014; 2(suppl 3): abstract P92.

66. Herzog T, Backes FJ, Copeland L, Estevez Diz MD, Hare TW, Huh W, et al. AIM2CERV: a randomized phase 3 study of adjuvant AXAL immunotherapy following chemoradiation in patients who have high-risk locally advanced cervical cancer (HRLACC). J Immunother Cancer. 2016:4(suppl 1): abstract P140.

67. Cohen EE, Moore KN, Slomovitz BM, Chung CH, Anderson ML, Morris SR, et al. Phase I/II study of ADXS11-001 or MEDI4736 immunotherapies alone and in combination, in patients with recurrent/metastatic cervical or human papillomavirus (HPV)-positive head and neck cancer. J Immunother Cancer. 2015;3(suppl 2): abstract P147.

68. Krupar R, Imai N, Miles B, Genden E, Misiukiewicz K, Saegner Y, et al. HPV E7 antigen-expressing Listeria-based immunotherapy (ADXS11-001) prior to 
robotic surgery for HPV-positive oropharyngeal cancer enhances HPVspecific T cell immunity. Cancer Res. 2016;76(14 suppl): abstract LB-095.

69. U.S. National Institutes of Health. ClinicalTrials.gov. Safety study of recombinant Listeria monocytogenes $(\mathrm{Lm})$ based vaccine virus vaccine to treat oropharyngeal cancer (REALISTIC). https://clinicaltrials.gov/ct2/show/ NCT01598792. Accessed 30 Nov 2016.

70. U.S. National Institutes of Health. ClinicalTrials.gov. A phase I/II evaluation of ADXS11-001, mitomycin, 5-fluorouracil (5-FU) and IMRT for anal cancer (276). https://clinicaltrials.gov/ct2/show/NCT01671488. Accessed 30 Nov 2016.

71. Fakih M, O'Neil BH, Chiorean EG, Hochster HS, Chan E, Mauro D, et al. Phase II study of ADXS11-001 in patients with persistent/recurrent, locoregional or metastatic squamous cell carcinoma of the anorectal canal. J Clin Oncol. 2016;34(4 suppl): abstract TPS786.

72. Guo T, Rettig E, Fakhry C. Understanding the impact of survival and human papillomavirus tumor status on timing of recurrence in oropharyngeal squamous cell carcinoma. Oral Oncol. 2016:52:97-103.

73. Chaturvedi AK, Engels EA, Pfeiffer RM, Hernandez BY, Xiao W, Kim E, et al. Human papillomavirus and rising oropharyngeal cancer incidence in the United States. J Clin Oncol. 2011;29:4294-301.

74. Gillison ML, Chaturvedi AK, Anderson WF, Fakhry C. Epidemiology of human papillomavirus-positive head and neck squamous cell carcinoma. J Clin Oncol. 2015;33:3235-42.

75. Geynisman DM, Chien CR, Smieliauskas F, Shen C, Tina Shih YC. Economic evaluation of therapeutic cancer vaccines and immunotherapy: A systematic review. Hum Vaccin Immunother. 2014;10(11):3415-24.

76. Darden PM, Thompson DM, Roberts JR, Hale JJ, Pope C, Naifeh M, et al. Reasons for not vaccinating adolescents: National Immunization Survey of Teens, 2008-2010. Pediatrics. 2013;131(4):645-51.

\section{Submit your next manuscript to BioMed Central and we will help you at every step:}

- We accept pre-submission inquiries

- Our selector tool helps you to find the most relevant journal

- We provide round the clock customer support

- Convenient online submission

- Thorough peer review

- Inclusion in PubMed and all major indexing services

- Maximum visibility for your research

Submit your manuscript at www.biomedcentral.com/submit
Biomed Central 